\title{
Sustainability factors for e-learning initiatives
}

\author{
Cathy Gunn*
}

Centre for Academic Development, The University of Auckland, Auckland, New Zealand

(Received 1 October 2009; final version received 28 April 2010)

\begin{abstract}
This paper examines the challenges that 'grass roots' e-learning initiatives face in trying to become sustainable. A cross-institutional study focused on local, rather than centrally driven, initiatives. A number of successful e-learning innovations were identified that had been driven by capable teachers seeking solutions to real educational problems. However, most of these remain in a high-risk category for institutions and offer poor rewards to their creators. While they may attract grant funding, these initiatives are typically not well supported in other important ways. Semi-structure interviews were conducted with 30 staff in various academic and support roles from the tertiary sector in New Zealand, who were nationally recognised as e-learning leaders. Analysis of their responses revealed both institutional and cultural barriers to long-term sustainability. The following influences on sustainability were identified and discussed: supportive organisational structures, a vision shared by all and staff accountability. The paper proposes that not all initiatives are sustainable and offer practical strategies for achieving diffusion and continuity through collective action. The paper concludes that there is a need for cross-functional collaboration to accommodate different and sometimes conflicting perspectives. This is just one aspect of the culture change that research shows is needed for e-learning to transform, rather than sit uncomfortably alongside, institutional practice.
\end{abstract}

Keywords: e-learning sustainability; e-learning strategy; integration; dissemination

\section{Introduction}

New Zealand (NZ) tertiary institutions, like their counterparts in other countries, pursue strategies to bring the pedagogical and practical benefits of e-learning to all staff and students. Enterprise-wide adoption of online learning management systems (LMS) is a common capacity-building strategy. Many locally driven teaching enhancement initiatives have also evolved to become an integral part of the e-learning environment. There is ample evidence that both strands of development enhance teaching and learning, yet the potential to transform practice is very different. The research described in this paper focuses on factors that challenge and enable long-term sustainability of smaller than enterprise-scale e-learning initiatives across the NZ tertiary sector. The literature identifies this as a common challenge across many years and national boundaries (see, for example, Darby 1992; Alessi 1997; Campbell 2003; O’Grady, Rouse, and Gunn 2009). 
The broad aim was to seek ways to improve the prospects for dissemination and long-term sustainability of quality e-learning systems that evolve from local teaching enhancement initiatives. The wisdom of supporting such initiatives is often questioned because of the complexity of supporting diverse e-learning systems. However, there is compelling evidence of the educational benefits they bring to host institutions and user communities. Local examples include reuse of complex learning designs (Gunn, Woodgate, and O'Grady 2005); transforming large class teaching through technology (Gunn and Harper 2007); enhancing learning through peer assessment (Hamer, Kell, and Spence 2007); and student-generated objective test item banks (Denny, LuxtonReilly, and Hamer 2008). With this evidence in mind, two assumptions underlie the study:

- While the e-learning initiatives in focus may not have potential to scale up to enterprise-wide application, the scope for their dissemination does warrant support, and the creative work of the enthusiasts that drive them deserves greater acknowledgement than it currently attracts;

- The investment of time, effort and resources in these initiatives could achieve greater educational and economic returns if appropriate forms of support were available. The lack of support creates high-risk situations that could be addressed for the benefit of all concerned.

The study was designed to inform local capacity development initiatives, where the persistent nature of common challenges demanded further investigation. A definition of sustainability is presented, and then the qualitative methodology and study details are described.

\section{Defining sustainability in the context of e-learning}

The term 'sustainable' has many possible interpretations. The definition offered here grounds the ensuing discussion in common understanding of the aims and outcomes of the research. An e-learning initiative is considered sustainable when all three of these conditions are met:

- A learning design involving information and communications technology has been developed and implemented within a course or courses of study. It has been through a proof-of-concept stage and has been judged, on the basis of evidence produced, to be beneficial to teaching and learning.

- The e-learning concept, design, system or resources have proven potential to be adopted, and possibly adapted, for use beyond the original development environment.

- Maintenance, use and further development of the e-learning concept, design, system or resources do not remain dependent on one or a few individuals who created them, to the extent that, if their involvement ceased, future prospects would not be compromised.

While other definitions are acknowledged, this one reflects a common scenario that challenges the aim to disseminate effective e-learning practices beyond the development context. Many institutional and nationally funded projects fall into this category, along with valuable work by early adopters (Taylor 1998). The overall aim of 
sustainable development is to transform teaching, learning and higher education organisations in the way described by Nicol and Draper (2009).

\section{Background}

The 'problem' of sustainability has been visible on the horizon for many years. During a nationally funded Flexible Learning Leaders in New Zealand initiative ${ }^{1}$ that ran from 2005 to 2007,3 individuals participated in a programme that required active involvement in flexible learning (used synonymously with e-learning in this paper), either as a lead practitioner or in a middle-management role. Group discussions revealed many challenges, including those that made dissemination and long-term sustainability of e-learning initiatives an elusive goal. A sub-set of these challenges was selected as the focus for further study, as described in this paper; that is:

- embedded institutional structures, policies and support systems that are at odds with evolving strategic goals for e-learning;

- the influence (or lack) of accountability measures on e-learning development, dissemination and uptake; and

- the effectiveness of strategies for maintenance, ongoing support, dissemination and to ensure continuity for successful e-learning systems and practices.

Formal study of these factors was considered useful for many reasons. Identifying barriers is the first step towards removing them. Framing deeper inquiry within an established research methodology supports publication of findings for discussion within and beyond the host institutions. Opening up the discussion locally could raise awareness and foster collaboration on developing solutions. It was assumed that networks and key relationships would provide a broad basis for problem-solving, dissemination and sharing of experience.

\section{Research aims}

The broad aim of the study was to use evidence from multiple cases to examine different perspectives on some common challenges to a key strand of e-learning integration at local, institutional and national levels. The focus was on 'grass roots' technologyenhanced teaching and learning initiatives that were devised to solve particular educational problems and were then recognised as having potential for use beyond the development context. Two outcomes were anticipated: greater understanding of a problem that continues to challenge the higher education sector after more than two decades, and practical ways to increase support for developers and other users of elearning innovations. A further aim was to improve a persistently low rate of return on investment of time, creative effort and scarce e-learning development resources.

\section{Methodology}

The study was grounded in a capacity development framework with two strands of activity: to identify and remove barriers to strategy implementation, and to explore and reconcile different stakeholder perspectives (Horton 2002). There is a known issue with gaps between policy and practice in educational change initiatives (Conole 
2008). Addressing this may be the key to success, as a report on one major area of elearning (e-portfolios) notes: "policies can drive forward an agenda for change, but the real test comes at the point of use" (Joint Information Systems Committee [JISC] 2008, 5).

Semi-structured interviews provided insight into the experience of e-learning practitioners, middle managers and academic support staff. This included 30 staff from six of New Zealand's eight universities and three polytechnics. The sample included five heads of academic development units, 10 e-learning support staff members (including librarians) and 15 lecturers who use a range of innovative e-learning strategies. The sample size and roles of participants supported investigation of different perspectives on common barriers. The assumption was that even a small-scale study would reveal common barriers and different views on how to address them.

The interview questions were broad (see Appendix 1) and the semi-structured format allowed different aspects to be brought to the fore, depending on the role and experience of the person being interviewed. Interviews lasted an hour and were more in the style of a conversation between colleagues than a formal interview.

\section{An opportunity for comparison}

Completion of the interviews coincided with publication of a report on a JISCsponsored project ${ }^{2}$ with similar aims. Guthrie, Griffiths, and Maron aimed to "gain systematic understanding of the mechanisms for pursuing sustainability" $(2008,4)$ and "to establish a context for conversation about further work in the area [of sustainability], and to prioritize areas of enquiry that would be helpful both to funders and to new projects" $(2008,5)$. While the JISC-sponsored work was broader in scope and conducted in a different national context, the aims and findings are similar to those of the NZ study in many important respects. This overlap is not surprising, as the NZ tertiary sector shares many characteristics with its overseas counterparts, and challenges to sustainable e-learning initiatives are known to be widespread. The opportunity to relate local experience directly to the broader international context was both timely and welcome.

\section{Findings}

The findings from this study confirmed that:

(1) sustainability of e-learning initiatives is a common challenge across the tertiary sector regardless of the scale and focus of the project;

(2) there is no single formula for sustainability that can be expected to work in every situation;

(3) strategies that have proved successful in different contexts are available for consideration; and

(4) accommodating different stakeholder perspectives is a critical success factor that needs to be interpreted with reference to specific contexts.

The first three points also feature in Guthrie, Griffiths, and Maron's (2008) report. The fourth is a key capacity development framework element that was foregrounded in the NZ study. Failure to acknowledge different perspectives may be a serious oversight, as very different experiences of the same initiative were revealed in the data. Other 
researchers have reached similar conclusions. For example, Lisewski talks about "the challenge of how the strategic approach will be translated into local academic cultures and practices on the ground" $(2004,184)$.

The challenges experienced at practice level can also be regarded as potential enablers of sustainability. They can be broadly categorised as the issues identified in the Background section above; that is, supportive organisation structures, including accountability measures, and strategies to achieve diffusion and continuity. The data revealed various ways in which these challenges arise in different institutional contexts. Findings are presented in summary format with illustrative quotes. To comply with the terms of ethics consent, these quotes are unattributed. The first area of focus is supportive organisational structures and processes.

\section{Supportive organisational structures}

Despite strategic intent to support teaching enhancement through e-learning, many instances were found where the gap between policy and practice was considerable.

I sort of wonder whether there should be more contestable funding ... of course the rewards for teaching initiatives are not great anyway.

Through a positive lens, the barriers identified in this category suggest the following enabling factors:

- an overall teaching and learning strategy that includes a vision for e-learning with accountability measures at both management and practitioner levels;

- flexible but measurable goals for e-learning that are reviewed on a regular basis with well-defined means of assessing performance against these goals; and

- a vision for e-learning that is relevant, coherent and shared.

If measurable goals and accountability processes are in place, then progress is both more visible and more likely to be supported. This may seem to state the obvious but many participants noted that these factors were not addressed in ways that enable progress on e-learning integration. The reasons are complex, although some common factors emerged.

\section{Is the vision really shared?}

The data showed that some institutions do not have an overall vision or strategy for elearning; and if they do, many people are unaware of it. This typically relegates development to the realm of enthusiasts who are prepared to work with low priority status and without institutional endorsement. Other institutions have strategies that focus exclusively on central initiatives such as the implementation of an enterprise LMS. In such cases, there may be no support for locally driven initiatives, regardless of how creative and educationally effective they are.

There have always been barriers of funding, and it turned out other people's definitions were much less about elearning than what I was doing. Many of the facilities that were put in place, I was already beyond. They couldn't offer me any help, what I really needed was a bit of money to develop my system. 
Only a few cases revealed strategies that offer practical support to grass-roots initiatives as well as centrally driven ones. Only one sought integration with existing organisational systems and processes.

We would love to have those facilities available on the standard lectern, so we just turn up with a memory stick or drag and drop our lecture from the LMS But, those kind of initiatives seem really slow. We've been pushing for years ... can we get this installed, can we get that installed. It's been quite frustrating that that hasn't changed.

Moves in this direction were found where, for example, information technology helpdesk staff were briefed to deal with staff and student support requests for new elearning systems. In the main, however, responsibility for dissemination and support remained with the developers. Since this is not the way central systems are managed, the message may come across that these are rogue systems. The belief that everything related to e-learning should be delivered by one enterprise system also led to a lack of support for many worthy initiatives.

The first year I got turned back by the internal committee. The second year, I thought what I need is collaboration, so I gathered together a 'who is who' in the discipline from several universities. I got cut back again.

This enterprise system focus has also spawned a flourishing range of work-around solutions to overcome limitations of system capability. If people have the skill to create solutions, it may be assumed that they also have skills to use the more convenient option of an enterprise system if it serves their purpose. Thus, the integration of emergent systems is happening in a haphazard way. The pace of change is also regarded as unacceptably slow.

The thing is with our department, if we see something and we think it's going to be great for our students and great for our teaching we want to run with it. We don't want to sit around and wait for a big study and a central decision so we'll use low cost or low-tech solutions if we can.

Two institutions reported information technology strategies designed to address these challenges through a web-service-oriented architecture approach. These works in progress will be watched with interest.

\section{Accountability as a driver of change}

Like students whose efforts are driven by assessment requirements, academics tend to be driven, to some extent, by accountability. Yet no cases were found where effective measures related directly to implementation of an e-learning strategy. As well as a means to monitor progress, accountability is just one way to acknowledge the considerable effort invested in e-learning development. While outcomes are reported to be intrinsically as well as educationally rewarding, no one considered e-learning development an easy road to travel. High workload, difficulty in finding resources, lack of real incentives to be creative and limited tangible rewards are common experiences. Supportive leaders are cited as a positive influence, although this tends to be based on personal relationships rather than determined by policy or institutional practice. To succeed, integration of e-learning requires the same conducive conditions, incentives and performance measures as any other strategic initiative. 
E-learning initiatives are acknowledged in performance review, promotion and awards schemes in some institutions. Accountability at senior levels is less common. This is seen as a negative influence on resource allocation and on perceptions of priority. Action is required to demonstrate the level of priority placed on e-learning development in a way that permeates an organisation. Performance indicators provide a structure to guide and measure achievement in the same way that rubrics guide students on assessment requirements. These indicators are context specific, although few examples were found.

\section{Accepting that not all initiatives are sustainable}

Some initiatives that succeed at the proof-of-concept stage fail to find the means of integration into an operational budget.

... we pulled the plug last December after two reasonably successful years. It never reached the point of critical mass for financial sustainability, so it became a drain on the University, on my department actually. I had a lot of support initially ...

In the case referred to above, an otherwise successful initiative stalled when fixedterm grant funding ran out. By that stage, it had been implemented in a number of faculties and outside the host institution. Four further cases showed the enthusiasm of developers waning under the pressure to achieve sustainability while managing other priorities. Factors that were perceived to be causing initiatives with acknowledged educational potential either to stall or to be abandoned include:

- pressure on individuals to maintain discipline-based research outputs rather than focus on teaching and learning enhancement;

- lack of access to ongoing funding after seed or fixed-term grants were used up;

- low levels of interest from colleagues and other potential users;

- developers with limited knowledge of dissemination strategies;

- staff changes resulting in loss of continuity; and

- lack of awareness or failure to use central services that could contribute to initiatives.

Collectively, these initiatives involved considerable creative effort, as well as many hundreds of hours devoted to funding applications, concept and materials development and evaluation. At the end of the day, investment through seed funding, teaching and learning grants and support services yielded little return. With strategic focus on e-learning, this situation is at best inefficient and at worst counterproductive. The question remains, is it the result of conscious decisions or simply a default scenario because no processes have been put into place to address it?

\section{Achieving diffusion and continuity}

The challenges to diffusion of successful e-learning innovations and to continuity for further development and future use have proved considerable in any circumstances (Stiles and Yorke 2006). This is exacerbated where individuals or small groups are the drivers. 
It is a model for what could be done, it is a model for getting teachers interested in using technology, for teachers looking at students results and informing classroom learning and all sorts of things.

Many academics report being too busy to prioritise exploring new approaches to teaching and learning, and there are cultural barriers to cross before engagement with successful initiatives developed elsewhere can occur. Various studies have attributed this phenomenon to the existence of academic tribes and territories (Becher and Trowler 2001), the need for a skill equivalent to cross-cultural communication (Gunn and Cavallari 2007) and the not invented here syndrome (Darby 1992).

Probably people are more willing to accept elearning stuff than they used to be but there is still a big barrier from the point of view of teachers adopting any thing that is new and different. I think the tide is gradually turning though.

While a degree of the same problem arises with centrally driven e-learning initiatives, the nature of the challenge is different. It is however, reasonable to suggest that strategies can be employed to address it, as the following scenario illustrates.

\section{Problem analysis and model solution}

Four separate cases highlighted the challenge of achieving diffusion and continuity, while a fifth offered a model of success. All five initiatives were developed to solve practical educational problems faced by teachers and have evolved to become mission critical elements of the learning environment over a number of years. Only one currently meets all the criteria for sustainability as defined in this paper, leaving four initiatives as high-risk scenarios for the institution and challenges with limited support for the developers.

The scenario is a common one, as changing circumstances, the pressures of scale and student diversity challenged teachers' ability to apply principles of good educational practice to course design. Capable teachers with some knowledge of the potential of e-learning and the skills and courage to experiment began to craft creative solutions to their respective problems. As development progressed and evidence of benefits to students emerged, the solutions were adopted by teachers in other disciplines. These cases all involve sophisticated forms of online assessment that allow students to engage with concepts, self-test, receive immediate constructive feedback and reflect on their learning. The learning designs required additional capabilities to those available through the enterprise LMS.

The basic tools that offers are great but we've found we need a lot of extra features. So for example our recorded lectures look to the student as if they're in the LMS, but they're actually not. There's no facility for us to upload them and keep everything formatted, so we just use a window of the LMS to link into our webpage.

An enterprise systems approach was being promoted within the host institutions during the early stages of these developments, so support was inconsistent. Grounding in the pedagogy of the discipline and responsiveness to student feedback were important design features, and the commitment of developers was undoubtedly a key success factor. In one case, the work had been in progress for 15 years at the time of the interview. 
As these initiatives grew and became visible, all but one was adapted for use beyond the development context, in some cases internationally. Student demand, collegial networks, publications and showcases of the work led other enthusiastic teachers to create content and activities for their courses. While the developers welcomed this endorsement of their work, it created new sustainability challenges. There are only so many new and continuing users that an individual with normal teaching and research commitments can manage. There is also a risk that staff changes will result in failure to capitalise on earlier investment in e-learning. Control of design and future direction make management by central services ('enterprisation') an unattractive prospect under current arrangements. The risk from the institutional perspective is that of reliance on individual developers for what has become an integral part of the learning environment in a number of teaching departments as well as in other institutions. A key area for action is to find ways to reduce these risks that are acceptable to all parties.

The one case that has become sustainable presents a model for success. This initiative pursued a team teaching approach from the outset, with tangible support from the head of department. This support took the form of resource allocation, workload accounting and recognition of teaching and learning enhancement initiatives in performance review and promotion processes.

We are rewarded, certainly within our department it's recognised as an important part of our work ... it's a core paper for the University so I think again it comes back to the HOD [head of department] and the senior members in the department who really do support us and see the value in putting time, people, money into building this course.

A course-specific suite of e-learning activities both includes and complements the enterprise LMS through seamless integration. The team teaching approach ensures continuity when staff changes occur. It also reaps the benefits of an internal community of professional practice, with more and less experienced academics working together on common goals. While no real effort has been made to disseminate this high-profile e-learning design beyond the development context, it has won highprofile teaching excellence awards, and the model is freely available for anyone to adopt or adapt. Initiatives such as this could feature as exemplars in institutional capacity development initiatives of the type described by Salmon, Jones, and Armellini (2008). However, most institutions do not analyse successful examples or use them to inform e-learning strategy implementation.

\section{Discussion}

Many barriers arise from the lack of support for e-learning innovation beyond that which is driven from the centre. While this is a deliberate strategy for some institutions, it is an unintended consequence for others. Some of those interviewed believed their experience exposed an institution paying lip service to statements of strategic intent around e-learning. It is equally likely to be a case of strategic intent confounded by limited grasp of the realities of implementation at practice level. Lisewski (2004) sums this up by saying that organisational cultural change needs to occur alongside the existence of key success parameters. He then warns against viewing organisational culture as simple or uniform and able to be shaped by managerial intentions.

It takes time for culture to change in what may be described as tradition-bound organisations with many levels of competing priorities. Furthermore, consensus 
reached during a roundtable discussion at the Association for Learning Technology Conference in 2009 was that policy generally follows after practice has established new ways of working in the area of e-learning. Since there are institutional leaders and managers who believe that grass-roots initiatives should be discouraged and only centrally driven ones supported, the risks and benefits of that approach may warrant investigation. However, many initiatives that featured in this study would have been stifled under that mandate, and there is absolutely no evidence to suggest that this would have been a good thing.

While some initiatives showed limited scope for diffusion early on, many with positive evaluation results and potential for wide dissemination were stifled by other factors. A proof-of-concept stage could determine viability in technical, financial and practical terms as well as educational ones. What happens after that is a situation that demands collective attention. The following sections raise questions for discussion and propose ways that the common barriers identified by this study might practically be addressed.

\section{Deciding where responsibility lies}

In their discussion of the impact of institutional culture on e-learning sustainability, Guthrie, Griffiths, and Maron (2008) note that an academic environment produces a particular mindset. They illustrate this by contrasting the role of a Principal Investigator for a research project with that of the leader of a sustainable business enterprise. A Principal Investigator is typically contracted to prove a concept and to develop and test it to the point of presenting a product and evaluation findings to the relevant community. Dissemination strategies are then applied to extend use of the product and findings beyond the development environment; that is, to address a key sustainability factor. The skills required for product development are not the same as those for promotion or dissemination, and the Principal Investigator or research team are not usually responsible for these later activities.

An easy parallel can be drawn with e-learning initiatives. Once a worthwhile product has been developed and evaluated, is it then fair to expect the developers to continue the drive for dissemination, maintenance and wider acceptance, or should processes be put in place to support these phases once the proof-of-concept phase is complete? To continue the comparison, a commercial enterprise has marketing, sales and promotion as separate functions to product design and development.

Despite noting the different skill set required, Guthrie, Griffiths, and Maron propose that the e-learning project leader should also be responsible for these later stages. In contrast, I argue that institutional systems should be put in place to support the output of the development phase. The rationale for this conclusion has many strands, including the drive for integration as a means to achieve sustainability. For example, institutional support networks could promote wider engagement, as well as acknowledging the achievement of the e-learning project team or individual. While questions about roles and responsibilities need urgent discussion, the answers probably lie in achieving a balance between the scale-focused priorities of central information technology services and the autonomy of teachers or developers to define the direction an emergent e-learning initiative will take. For this to succeed, different perspectives clearly need to be explored and most probably reconciled to align with strategic priorities. The question that remains is, who is responsible for raising these questions and driving the discussion at institutional level? The problem in many cases 
is that no one considers it their responsibility and that lead practitioners have the experience but not necessarily the power or the will to influence senior management thinking.

\section{Avoiding high-risk situations}

One of the main arguments against supporting grass-roots e-learning initiatives is the high-risk situation that can result from their success. The study identified four cases where systems had become an integral part of the learning environment, yet staffing, resource allocation and support arrangements remained tenuous.

When I started getting external funding, the amount of support from the University went down by about a third. I now get about a third from the University, but that is under threat every single year.

Rather than withdrawing support from initiatives that are clearly feasible and successful in educational terms, another approach would be to reconfigure central services to offer greater flexibility and stability. There are many cases where this would serve the interests of staff and students, as well as aligning with e-learning strategies.

What has kept me going, the whole time, and what I also think the University ultimately can't ignore is the response from the students to what I'm doing.

Rather than initiatives having to fit within existing funding and support structures, a more flexible approach could offer different options in response to demand. A mandate for central services to work with grass-roots initiatives would do much to lower the risk. The need in this case is for greater flexibility, assistance with proof of concept and practical strategies to meet dissemination, maintenance and support requirements of successful initiatives. Collaboration is a key factor that represents a culture shift in most cases.

\section{The need for collective action}

The various roles of participants in the study reflect an emergent trend towards crossfunctional relationships around e-learning. Barriers to sustainability have been clearly and repeatedly identified, yet action to effect their removal remains ill-defined and dependent on uncommon configurations for collective action. Discussion amongst key players in e-learning strategy implementation is needed to define the nature of that action and to identify appropriate lines of responsibility and accountability measures. The Guthrie, Griffiths, and Maron (2008) report's different view on these matters demands further discussion, and may point to complementary strands of action rather than conflicting opinions on a solution to the complex problem of sustainability. The report's failure to acknowledge different stakeholder perspectives may be a limiting factor on the value of its recommendations. However, the question it poses is a valid one.

Clearly the leaders of these [e-learning] initiatives are competent professionals; why do they not rely on processes that have proven effective in both commercial and not-forprofit contexts? (Guthrie, Griffiths, and Maron 2008, 5) 
It is reasonable to suggest that this approach would have worked by now if it was going to. It is also acknowledged that strategic initiatives driven from the centre or top levels of an organisation often fail to appreciate the impact at practice level. Similarly, recommendations that fail to acknowledge various impact factors may also fail to address critical success factors, or to value the wisdom gained through front-line experience.

On the question of whether or not sole responsibility for driving design, development and dissemination processes should be left to the individual with the original good idea, I conclude that it is both unreasonable and inefficient to expect this model to succeed in institutional environments that promote, and even demand, collaboration. Support for a low-risk proof-of-concept phase followed by collective action to disseminate and maintain could both streamline and bring useful expertise to the process.

\section{Conclusions}

Questions about e-learning sustainability arise in many contexts. One example is New Zealand's national Flexible Learning Leaders Project, which focused on development of individuals as e-learning leaders. Challenges faced in the course of these leaders' professional practice included many related to long-term sustainability of the initiatives in which they were involved. Research questions for the study described in this paper thus arose from real professional practice contexts in authentic institutional settings. The current study drew on the literature, gathered data to explore the experience of various e-learning initiatives and examined different perspectives on problems and possible solutions to the challenges of sustainability. The study was representative of a national sector that has a total of just eight universities. The findings offer a broad view of current challenges to e-learning sustainability across the sector. Similar challenges are reported in other national contexts, suggesting the questions addressed are broadly applicable and the findings have potential to generalise. Discussion of these issues is invited, and additional data focusing on challenges at professional practice level will be the subject of a separate publication.

To conclude this analysis of institutional issues, the benefits as well as the challenges of supporting sustainable e-learning initiatives beyond an enterprise LMS have been demonstrated. While the spirit of many e-learning strategies promotes this kind of diversity, institutional systems and practices still need to align to make this support tangible and effective. Evidence shows that the shared vision and sense of ownership required for successful strategy implementation is more likely to arise where such diversity is encouraged. The flexibility to meet a variety of user needs is recommended and a greater range of creative and pedagogically driven initiatives is likely to result. Collective action is needed to make this flexible approach more stable and sustainable than it currently is. This demands an uncommon degree of cross-functional collaboration to accommodate different and sometimes conflicting perspectives. This is just one aspect of the culture change that research shows is needed for e-learning to transform, rather than sit uncomfortably alongside, institutional practice.

E-learning challenges many embedded traditions, which will take time and targeted action at different institutional levels to shift. Willingness to work with a new collaborative model to devise and implement strategy, as well as for e-learning development at practice level, is a particularly challenging target in institutions with 
long-standing traditions of hierarchy. In fact, what is required is a collective rather than a collaborative approach to streamline institutional response times and policy development processes. This will doubtless encounter as many design and implementation challenges as the e-learning initiatives it is intended to serve. Evidence to support the wisdom of this approach has been gathered from various sources over the past two decades. The challenges that remain are to craft a successful solution to remove barriers to sustainability, and to determine where responsibility for driving it actually lies. One barrier may be that this responsibility is no longer exclusively in the hands of powerful individual leaders as the knowledge and experience gained at practice level has proved essential to good strategic process. Consultation across institutional levels is needed to allow such knowledge to inform strategic initiatives. This involves a different mindset and new institutional systems that offer tangible support to educationally sound 'grass roots' e-learning initiatives. A balance needs to be struck between standardisation and central control on the one hand, and freedom to experiment and choose on the other. While this may sound simple on one level, it often requires power shifts and establishment of new roles and functional relationships, which can be challenging to achieve. The value of many local e-learning initiatives has been proved by their survival in environments that typically favoured centrally driven solutions. A supportive environment could only help more of them to flourish, and allow educational design to drive the strategic e-learning agenda.

\section{Notes}

1. Flexible Learning Leaders in New Zealand initiative. http://www.verso.co.nz/flinzflexible-learning-leaders-in-new-zealand/.

2. The UK-based JISC is a national organisation supporting leadership in the use of information and communications technology in educational settings.

\section{References}

Alessi, S. 1997. Seeking common ground: Our conflicting viewpoints about learning and technology. Instructional Technology Research Online. http://www2.gsu.edu/ wwwitr/ docs/common/index.html.

Becher, T., and P. Trowler. 2001. Academic tribes and territories. Milton Keynes: Open University Press.

Campbell, L. 2003. Engaging with the learning object economy. In Reusing online resources: A sustainable approach to elearning, ed. A. Littlejohn, 35-45. London: Kogan Page.

Conole, G. 2008. New schemas for mapping pedagogies and technologies. Ariadne 56. http:// www.ariadne.ac.uk/issue56/conole/.

Darby, J. 1992. The future of computers in teaching and learning. Computers and Education 19, nos. 1-2: 193-7.

Denny, P., A. Luxton-Reilly, and J. Hamer. 2008. The PeerWise system of student contributed assessment questions. In Proceedings of the tenth conference on Australasian Computing Education, Conferences in Research and Practice in Information Technology 8, ed. M. Hamilton, 69-74. Wollongong, NSW: Australian Computer Society.

Gunn, C., and B. Cavallari. 2007. Instructional design, development and context expertise: A model for 'cross cultural' collaboration. In Instructional design: Case studies in communities of practice, ed. M. Keppel, 127-50. New York: IGI Global.

Gunn, C., and A. Harper. 2007. Using elearning to transform large class teaching. In Making the transition to elearning: Issues and strategies, ed. M. Bullen and D. Janes, 139-56. Hershey, PA: IGI Global.

Gunn, C., S. Woodgate, and W. O'Grady. 2005. Repurposing learning objects: A sustainable alternative? ALT-J Research in Learning Technology 13, no. 2: 189-200. 
Guthrie, K., R. Griffiths, and N. Maron. 2008. Sustainability and revenue models for online academic resources: An Ithaka report. Bristol, UK: JISC/Strategic Content Alliance.

Hamer, J., C. Kell, and F. Spence. 2007. Peer assessment using Aropa. In Proceedings of the Ninth Australasian Computing Education Conference (ACE 2007), ed. S Mann, 43-54. Ballarat, VIC: Australian Computer Society.

Horton, D. 2002. Planning, implementing and evaluating capacity development. The Hague: International Service for National Agricultural Research.

Joint Information Systems Committee. 2008. Effective practice with eportfolios. http:// www.jisc.ac.uk/publications/publications/effectivepracticeeportfolios.aspx.

Lisewski, B. 2004. Implementing a learning technology strategy: Top down strategy meets bottom up culture. ALT-J Research in Learning Technology 12, no. 2: 175-88.

Nicol, D., and S. Draper. 2009. A blueprint for transformational organizational change in higher education: REAP as a case study. In Education through technology-enhanced learning, ed. T. Mayes, D. Morrison, H. Mellar, P. Bullen, and M. Oliver, 191-207. York: The Higher Education Academy.

O'Grady, W., P. Rouse, and C. Gunn. 2009. Synthesizing management control frameworks. Paper presented at the annual conference of the Performance Measurement Association, April 14-17, in Dunedin, New Zealand.

Salmon, G., S. Jones, and A. Armellini. 2008. Building institutional capability in e-learning. ALT-J Research in Learning Technology 16, no. 2: 95-110.

Stiles, M., and J. Yorke. 2006. Technology supported learning - tensions between innovation, and control and organizational and professional cultures. Journal of Organizational Transformation and Social Change 3, no. 3: 251-67.

Taylor, P. 1998. Institutional change in uncertain times: Lone ranging is not enough. Studies in Higher Education 23, no. 3: 269-79. 


\section{Appendix 1. Interview questions about e-learning initiatives}

1) What led you to become involved in elearning initiatives initially?

2) Focusing on a key initiative, can you give a brief outline of the concept and how you developed it as a learning design or learning resource?

3) Or b) Generally speaking, how have you developed elearning concepts into learning designs or resources?

4) What special skills did you need to accomplish this, and did you have access to all the skills you needed, either personally or from outside sources? What sources?

5) Can you describe an ideal application or use scenario?

6) What do you consider to be the main benefits to learners?

7) Are there also specific benefits for teachers, and if so, what are they?

8) Is/are the elearning initiative/s you have been involved in an integral part of yours or others' discipline based teaching responsibility?

9) What would encourage you to continue to be involved in elearning development, and what resources and support [would] make that possible?

10) Is there any potential, or has the initiative moved outside the development context or discipline? Is so, how did or could this happen?

11) Can you describe any major barriers or challenges you faced during the development, implementation and dissemination processes?

12) Have you ever considered commercialization as a way to support ongoing development?

13) Do you think your department and the institution have suitably acknowledged the time and effort you put into this initiative?

14) How would you set up an ideal situation for others going down this path?

15) Do you think institutions could play a greater role in dissemination and support for initiatives of this kind? 